\title{
Shaping Bipolar and Elliptical Planetary Nebulae: Rotation and Magnetic Field
}

\author{
Guillermo García-Segura ${ }^{1}$, Norbert Langer ${ }^{2}$, Michał Różyczka ${ }^{3}$, \\ Mordechai-Mark Mac Low ${ }^{4}$ and José Franco ${ }^{1}$ \\ ${ }^{1}$ Instituto de Astronomía-UNAM; ${ }^{2}$ Max-Planck-Institut für Astrophysik; \\ ${ }^{3}$ Warsaw University Observatory; ${ }^{4}$ Max-Planck-Institut für Astronomie
}

We present hydrodynamical and magnetohydronynamical simulations for the formation and evolution of bipolar and elliptical planetary nebulae with two interacting winds. The models are performed under the hypothesis of a single central source, i.e. binary systems are not considered and a single initial wind function is used in our calculations. We explore various relevant parameters, including the effects of stellar rotation, ionizing radiation field and stellar magnetic field, and a catalogue of resulting shapes is generated.

Our main result is that rotation in the non-magnetic case can generate bipolar shapes, whereas the inclusion of the stellar B-field can account for the rest of the observed morphologies.

We find that strongly bipolar nebulae can be obtained from single stars when the stellar rotational velocity, $v_{\text {rot }}$, approaches the critical rotational velocity, $v_{\text {crit }}$, at the asymptotic giant branch (AGB) phase. As an example, the shape of the "hour glass nebula" (MyCn18) is obtained for the value of the ratio $\Omega=v_{\text {rot }} / v_{\text {crit }} \approx 0.98$.

The classical elliptical shapes such as IC 4593 can be achieved when the ratio of the magnetic field energy density to the kinetic energy density, $\sigma$, in the fast wind is close to the actual Solar value, $\sigma \simeq 0.009$, even for spherical AGB winds. When the stellar magnetic field is combined with the effect of the rotation at the AGB phase, highly collimated bipolar nebulae can be obtained such as M2-9 or He 2-437. An important effect of the stellar magnetic field is the formation of ansae and jets in the polar regions because of the magnetic tension.

Photoionization is also important in producing dynamical effects. It generates a series of irregularities in the shape of the simulated nebulae, and is probably responsible for the formation of the irregular planetary nebula type such as Sh 2-71. It leads to the formation of cometary knots, preferentially at the equatorial region, as the one seen in the Helix nebula (NGC 7293). 